\title{
Visual feedback information for virtual lifting tasks
}

\begin{abstract}
This paper investigates the effect of visual feedback techniques to enhance user performance in virtual environments (VE) while carrying out manual lifting tasks. The aim of this study was to reduce lower back pain by enabling users to monitor their lower back condition in terms of lifting index (LI) values, whilst moving an object from one location to another. Several experiments were conducted to explore and evaluate the effectiveness of neutral, visual and combined visual feedback on lifting performance. An analysis of task completion time, percentage of harmful lifts and response time to feedback were analysed and used to identify the appropriateness of each visual feedback technique. The results showed that the addition of visual feedback did introduce an improvement in manual lifting tasks. The results also reveal that the combined visual feedback techniques performed better than singular feedback techniques.
\end{abstract}

Keyword: Visual feedback; Lower back pain; Lifting 